\title{
A Huge Morgagni Hernia in a 62-Years-Old Female Patient
}

\author{
Ali Naki Yücesoy \\ Clinic of Emergency Medicine, Batı Bahat Hospital, İstanbul, Turkey
}

A 65-years-old female patient was admitted to our emergeny department with severe intermittent epigastric pain, which radiated to the right thoracic region. She had an ongoing intermittent epigastric pain since a week. On physical examination, decreased breath sounds in the right hemithorax and epigastric tenderness were found. Laboratory examination detected no abnormal findings except mild leukocytosis. On plain chest roentgenogram, a large soft tissue mass containing a portion of opacified colonic structure filled with the remnant of a previously performed barium enema was observed in the right hemithorax (Figure 1). A thoracoabdominal CT revealed a herniated colonic segment and omentum in the right hemithorax. The patient underwent surgery, and an ischemic herniated transverse colonic segment and omentum, which passed to the right hemithorax through the right anterior sternocostal defect (Morgagni's hernia) were detected. After reduction of the herniated colonic segment and omentum, the diaphragmatic hernia was repaired using prolene mesh.

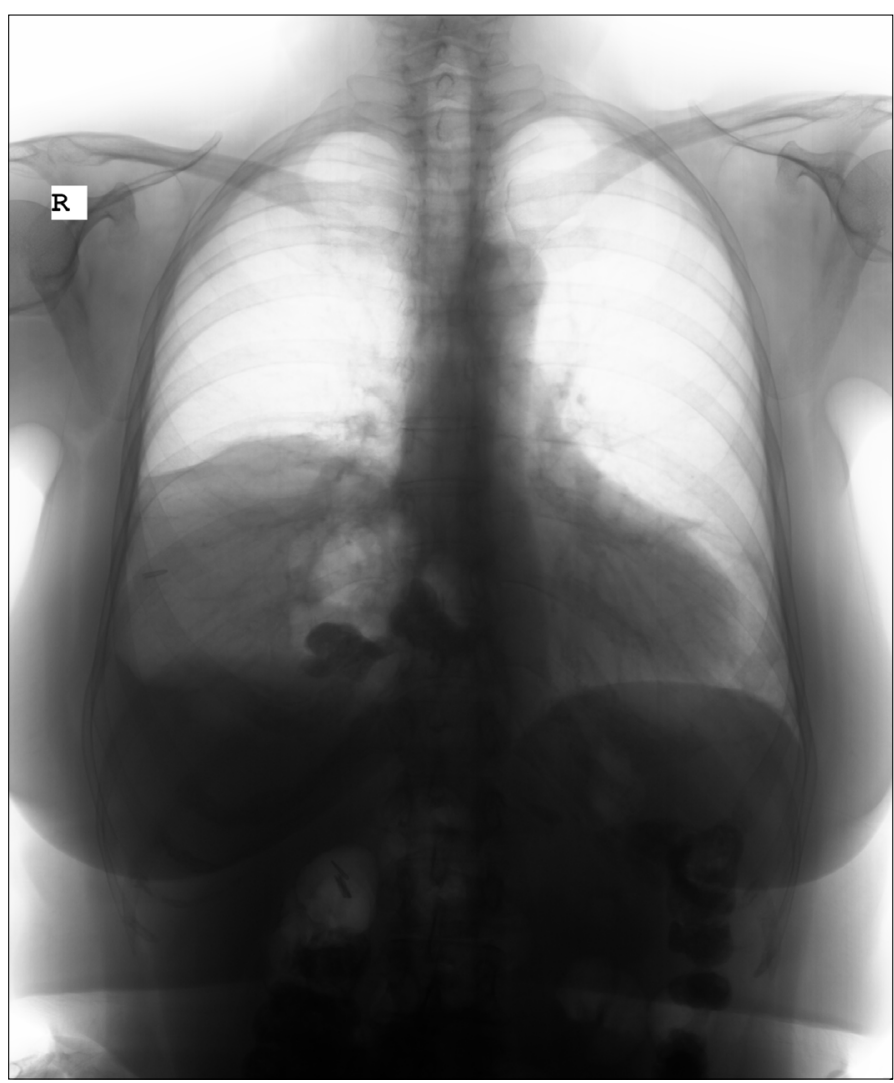

Figure 1. An image of the huge Morgagni's hernia on plain chest roentgenogram 


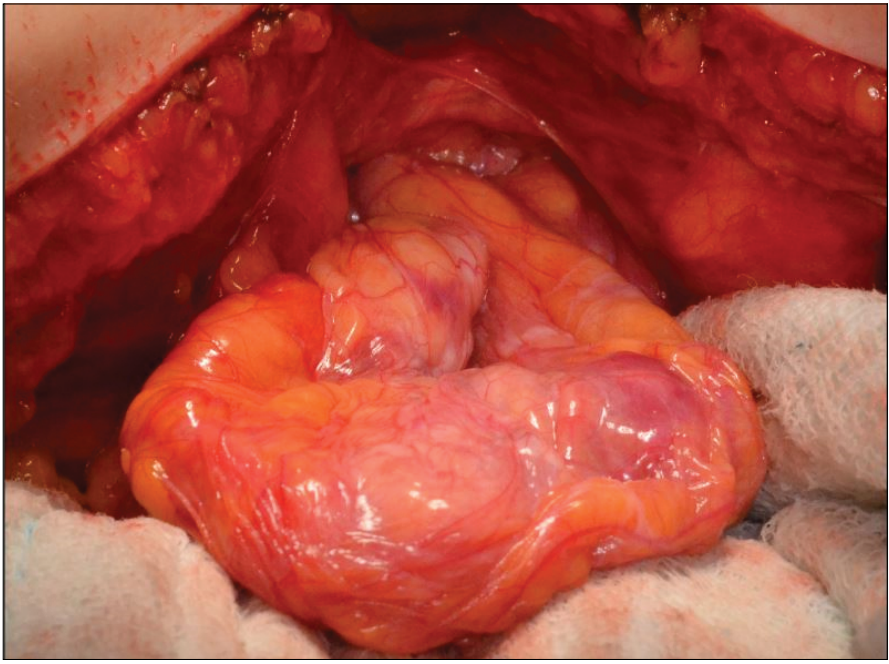

Figure 2. A view of the huge Morgagni's hernia reduced into the abdominal cavity

\section{Discussion}

Morgagni's hernia can be defined as the protrusion of any abdominal content into the thoracic cavity through the congenital anterior sternocostal defect. Morgagni's hernia was first described by the Italian anatomist and pathologist Morgagni in 1769 (1). Morgagni's hernia is a rare condition and constitutes approximately $2 \%$ $3 \%$ of congenital diaphragmatic hernias. It is usually found on the right side of the diaphragm and rarely on the left (2). Hernial defect repair without removing hernial sac is the most common surgical procedure, which can be performed via abdominal, thoracic, or laparoscopic approaches. Although Morgagni's hernia is a congenital diaphragmatic hernia, it is rarely seen in adults. Pulmonary and epigastric complaints are the most common symptoms in adult Morgagni's hernia cases, which can be complicated by strangulation or obstruction (3). In our case, strangulation findings recovered with reduction were observed in the herniated transverse colon segment. Adult Morgagni's hernia is a very rare condition, and immediate surgical intervention may be required in some cases.

\section{References}

1. Morgagni GB. The seats and causes of diseases investigated by anatomy. Vol. 3. London: Miller and Cadell 1769. p. 205.

2. Minneci PC, Deans KJ, Kim P, Mathisen DJ. Foramen of Morgagni hernia: changes in diagnosis and treatment. Ann Thorac Surg 2004; 77: 195-9. [CrossRef]

3. Horton JD, Hofmann LJ, Hetz SP. Presentation and management of Morgagni hernias in adults: a review of 298 cases. Surg Endosc 2008; 22: 1413-20. [CrossRef] 